\begin{tabular}{|c|c|}
\hline Title & $\begin{array}{l}\text { A nalysis of Leakage Losses in One Dimensional Photonic Crystal Coupled Resonator O ptical Waveguide Using 3-D } \\
\text { Finite Element Method }\end{array}$ \\
\hline Author(s) & Kawaguchi, Y uki; Saitoh, Kunimasa; Koshiba, Masanori \\
\hline Citation & $\begin{array}{l}\text { Journal of Lightwave Technology, 28(20), 2977-2983 } \\
\text { https://doi.org/10.1109/JLT.2010.2074185 }\end{array}$ \\
\hline Issue Date & 2010-10-15 \\
\hline Doc URL & http:/hdl. handle.net/2115/44162 \\
\hline Rights & $\begin{array}{l}\text { () } 2010 \text { IEEE. Personal use of this material is permitted. However, permission to reprint/republish this material for } \\
\text { advertising or promotional purposes or for creating new collective works for resale or redistribution to servers or lists, } \\
\text { or to reuse any copyrighted component of this work in other works must be obtained from the IEEE. }\end{array}$ \\
\hline Type & article \\
\hline File Information & JLT 28-20_2977-2983.pdf \\
\hline
\end{tabular}

Instructions for use 


\title{
Analysis of Leakage Losses in One-Dimensional Photonic Crystal Coupled Resonator Optical Waveguide Using 3-D Finite Element Method
}

\author{
Yuki Kawaguchi, Student Member, IEEE, Kunimasa Saitoh, Member, IEEE, and Masanori Koshiba, Fellow, IEEE
}

\begin{abstract}
We have evaluated leakage losses of one-dimensional photonic crystal coupled resonator optical waveguides (1-D PC-CROW) by using three-dimensional finite element method (3-D FEM). We propose structure of 1-D PC-CROW with shifted position of air-hole, changed size of air-hole, and changed air-hole depth. We have also proposed air-bridge 1-D PC-CROW and evaluated group velocity and leakage losses of these CROWs. Our numerical results show that 1-D PC-CROW has large leakage losses, especially for vertical direction, and the proposed structure can reduce leakage losses. Leakage loss of 1-D PC-CROW with changed air-hole depth is two order of magnitude lower compared with normal structure.
\end{abstract}

Index Terms-Coupled resonator optical waveguides, finite element method, leakage loss, photonic crystals.

\section{INTRODUCTION}

$\mathbf{C}$ OUPLED RESONATOR OPTICAL WAVEGUIDES (CROWs) are highly attractive for integrated delay lines [1]-[3] and nonlinear effects enhancement [4]. Especially, optical delay lines have emerged as key components for future optical networks and information processing systems. There is a wide range of applications of optical delay lines, such as optical buffers [5], optical regenerators [6], wavelength converters [7], and optical delay-line filters [8]. CROWs are attractive for these applications because of their specific small group velocity and zero group velocity dispersion (GVD) at the transmission miniband [9]-[19]. CROW can be classified in several categories in terms of the resonance structures such as, CROW relying on ring resonators [18], Fabry-Perot cavities, and photonic crystal (PC) microcavities [12], [13], [15], [16], [20]. Among configurations relying on one- to three-dimensional PC cavities, one-dimensional (1-D) PC-CROWs based on photonic wires are the most attractive in terms of simplicity, while keeping the advantages of PCs such as compactness and integrability [21], [22]. Such 1-D PC-CROWs are also useful with respect to experimental applications because 1-D PC-CROW is composed by cascading 1-D PC microcavities and

Manuscript received July 22, 2010; revised August 30, 2010; accepted August 31, 2010. Date of publication September 09, 2010; date of current version October 04, 2010. The work of Y. Kawaguchi was supported by the Japan Society for the Promotion of Science.

The authors are with the Graduate School of Information Science and Technology, Hokkaido University, Sapporo 060-0814, Japan (e-mail: kawaguchi@icp.ist.hokudai.ac.jp).

Color versions of one or more of the figures in this paper are available online at http://ieeexplore.ieee.org.

Digital Object Identifier 10.1109/JLT.2010.2074185 there are a lot of reports of 1-D PC microcavities [23], [24]. One of the drawbacks of 1-D PC-CROW is its intrinsic propagation losses due to diffraction. This strongly limits their usefulness in various applications [25]. However, recently we have shown a loss reduction mechanism for the 1-D PC-CROW and low-loss 1-D PC-CROW structure, therefore the low-diffraction-loss 1-D PC-CROW can be a good candidate for developing various ultra compact optical circuits, while a uniform structure for the vertical direction has been assumed in the previous work [26]. For a practical application, we need to design optical devices considering leakage losses of both horizontal and vertical direction. However, there are no reports that evaluate them quantitatively. Therefore, in this work, we have evaluated dispersion relationships and leakage losses of 1-D PC-CROW in details by using three-dimensional finite element method (FEM) for periodic structure analysis [27].

The remainder of this paper is structured as follows. In Section 2, we describe a model and fundamental characteristics of 1-D PC-CROW. We show that leakage losses of the vertical direction is quite high and 3-D analysis is important to examine waveguide characteristics of 1-D PC-CROW. In Section 3, we characterize four types of 1-D PC-CROW to achieve low-loss structure, 1-D PC-CROW with shifted air-hole or changed air-hole size, air-bridge 1-D PC-CROW, and 1-D PC-CROW with shallow etched air-hole. In Section 4, findings in the numerical analysis are summarized.

\section{STRUCTURE OF NORMAL 1-D PC-CROW}

We consider a 1-D PC-CROW as shown in Fig. 1. We set structural parameters as follows. The lattice constant $a$ of 1-D PC is $400 \mathrm{~nm}$, the waveguide width $w$ is $1.2 a=480 \mathrm{~nm}$, the air hole radius $r$ is $0.3 a=120 \mathrm{~nm}$, the distance between air holes across the defect $b$ is $2.5 a=1000 \mathrm{~nm}$ [26], and the waveguide height $h$ is $0.5 a=200 \mathrm{~nm}$. In this case, the intercavity distance $\Lambda$ becomes $5.5 a=2200 \mathrm{~nm}$. We assume silicon, silica, and air as core, under cladding, and over cladding materials, respectively. We set the refractive index of 3.5 as silicon and 1.45 as silica.

In Figs. 2(a) and (b), we show the dispersion curve and the group velocity in 1-D CROW for quasi-TE mode calculated by 3-D FEM for periodic structure waveguides [27] with solid curves, where $\lambda$ is the operating wavelength, $\beta$ is the propagation constant along the propagation direction ( $z$ direction), $v_{g}$ is the group velocity, and $c$ is the velocity of light in vacuum. Here we give some details about numerical implementation. The analysis region is divided into linear tangential/quadratic normal 


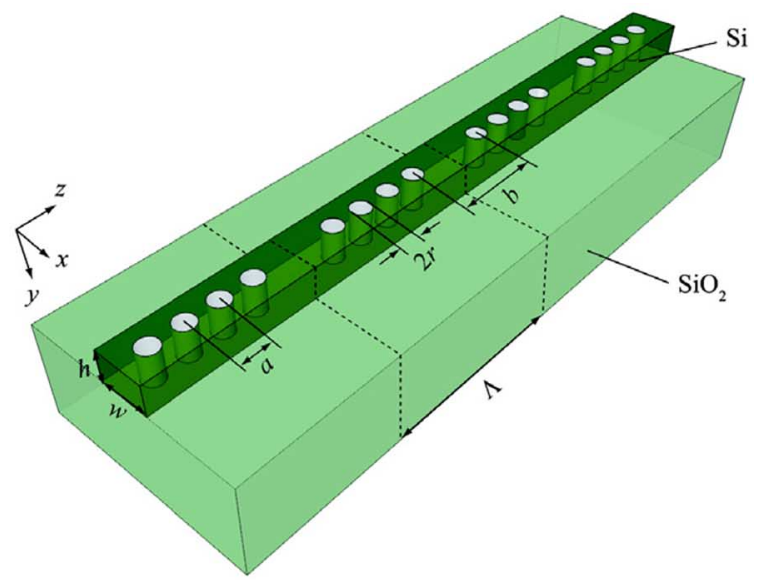

(a)



(b)

Fig. 1. (a) Top view of 1-D PC-CROW and (b) enlarged view for one period.

(LT/QN) tetrahedral element (20 variables). The size of each element needs to be carefully chosen in order to converge the solution. We confirmed the convergence and divided the analysis region. The number of nodes and edge variables used for the calculation are 162625 and 745876 , respectively. In order to solve the eigenvalue equation, the method of inverse iteration with shifts of origin is applied. The matrix equations to be solved in this iterative procedure are sparse complex unsymmetric linear systems of equations, which can be solved with appropriate software, such as PARDISO library [28]. We also plot dispersion curve and group velocity calculated by 2-D FEM [26] with dashed curve for comparison. Structural parameters for 2-D analysis are the same with 3-D analysis except for $h$. Since a uniform structure is assumed for $y$ direction, waveguide height is set as $h=\infty$. The transmission miniband of the guided mode allocated within the PBG exists from the normalized frequency $\Lambda / \lambda$ of 1.437 to 1.469 and the zero GVD is obtained around the central frequency of the miniband. In the zero GVD region, the normalized group velocity $v_{g} / c$ is about 0.095 . Compared with 2-D analysis, dispersion curve shifts to higher frequency and group velocity is almost same value. In this 2-D calculation, the refractive index of core material is set as 3.5. Of course, the operating wavelength gets close to 3-D simulation results by applying effective index method. However, we note that when we adopt effective index method, group velocity becomes larger because the strength of refractive index modulation changes. From the result of group velocity $v_{g}$ in Fig. 2(b), we can evaluate propagation losses as follows. In the used FEM formulation, a complex frequency of the guided mode is obtained as an eigenvalue from a predefined propagation constant $\operatorname{Re}(\beta)$ by solving a complex eigenvalue problem, where $\operatorname{Re}(\beta)$ stands for the real part of the propagation constant. Then we can obtain the imaginary part of the propagation constant $\operatorname{Im}(\beta)$ by the following equation [29]

$$
\operatorname{Im}(\beta)=\operatorname{Im}(\omega) / v_{g}
$$

where $\omega$ is the angular frequency and the leakage loss can be estimated as 8.686| $\operatorname{Im}(\beta) \mid$. In Fig. 2(c), we show the leakage losses as a function of the normalized frequency. The leakage loss becomes small around the zero GVD region and the loss is $0.5 \mathrm{~dB}$ per one period. Dashed curve in Fig. 2(c) shows leakage loss of 2-D analysis. Leakage losses of 1-D PC-CROW, which is assumed to be a uniform structure in the $y$ direction, is about $0.07 \mathrm{~dB}$ per period. From this result, we can see that leakage loss for the vertical direction is quite high and 3-D analysis is absolutely imperative for examining transmission characteristics of 1-D PC-CROW.

\section{DeSIGN OF LOW-LOSS 1-D PC-CROW}

\section{A. 1-D PC-CROWs With Shifted Air-Holes}

Next, we address to reducing the leakage losses in 1-D PC-CROW. If the reflection of the electromagnetic field is weakened at the cavity edge, the change of electromagnetic field becomes gentler and its envelope function gets close to Gaussian function. As a result, the tangential components of wavevector in the leaky region are reduced, leading to the suppression of leakage losses [26]. In order to weaken the reflection of the electromagnetic field at the cavity edge, we shift two air holes' position neighboring cavities to outside as shown in Fig. 3(a), where the displacement of the air holes at the cavity edges is set as $S$. In Figs. 3(b), (c), and (d), we show dispersion curves, group velocity, and leakage losses, respectively, where $S$ is a variable parameters. By increasing the displacement of the air holes, dispersion curve shifts to lower frequency side and group velocity becomes large. As was expected, the leakage losses are reduced to $0.4 \mathrm{~dB}$ per period at the central wavelength, however, the suppression of the leakage losses is not enough.

\section{B. 1-D PC-CROW With Changed Air-Hole-Size}

As another approach to weaken electromagnetic field reflection at the cavity edges, we consider changing two air holes' radius neighboring cavities $r$ to $r^{\prime}$ as shown in Fig. 4(a). In Figs. 4(b), (c), and (d), we show dispersion curves, group velocity, and leakage losses, respectively, where the modified airhole radius is set as $r^{\prime}=0.3 a(=r), 0.25 a$, and $0.2 a$. As in the case of previous approach, the dispersion curve shifts to lower frequency side and group velocity becomes large by decreasing the air-hole radius. We can see that the leakage losses are reduced by adjusting two air holes' radius neighboring cavities. Specifically, when we set $r^{\prime}=0.2 a$, the leakage losses are reduced to about $0.2 \mathrm{~dB}$ per period at the central wavelength. 


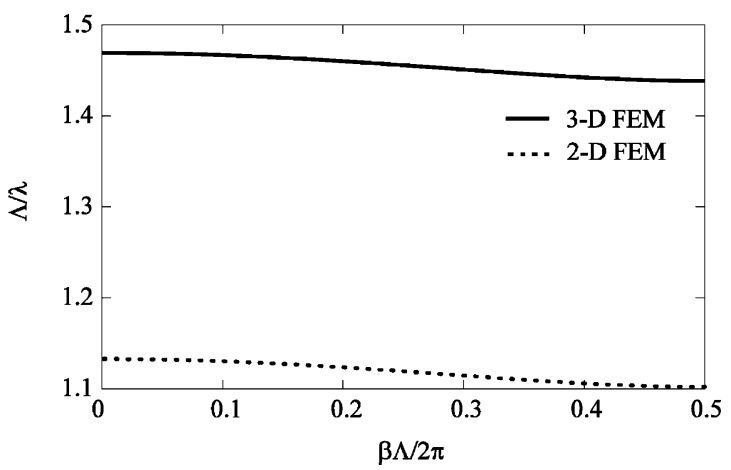

(a)

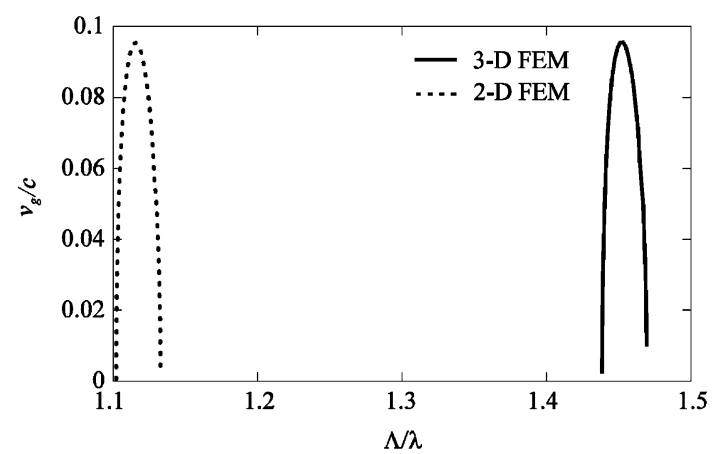

(b)

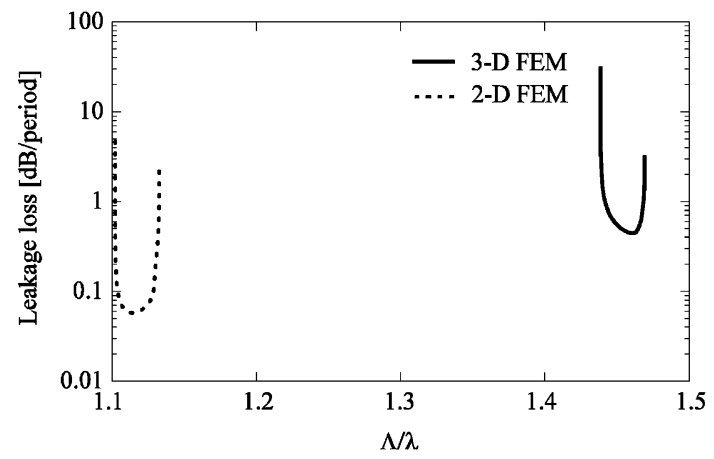

(c)

Fig. 2. (a) Dispersion curve, (b) group velocity, and (c) leakage loss of 1-D PC-CROW.

In this structure, calculated normalized group velocity $v_{g} / c$ is about 0.13 .

\section{Air-Bridge 1-D PC-CROW}

We consider weakening the reflection of electromagnetic field at the cavity edge to reduce the leakage losses so far, however, they need to be decreased further for practical applications. For designing low-loss 1-D PC-CROW structure, we consider another approach for reducing leakage losses. We can expect that leakage losses for vertical direction are suppressed by making relative refractive index difference higher, therefore, next we evaluate leakage losses of air-bridge 1-D PC-CROW as shown in Fig. 5(a). Red curves in Figs. 5(b), (c), and (d) show dispersion curve, group velocity, and leakage loss, respectively, for the air-bridge structure $\left(r^{\prime}=0.3 a\right)$. We can see that the dispersion curve of air-bridge 1-D PC-CROW shifts to higher frequency region. And we can also see that the group velocity becomes smaller. This is because air-bridge structure can confine

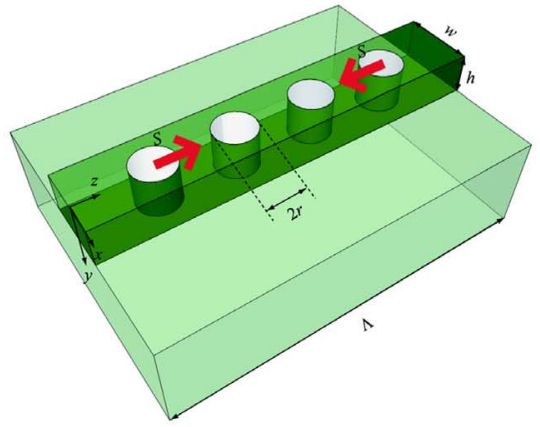

(a)



(b)

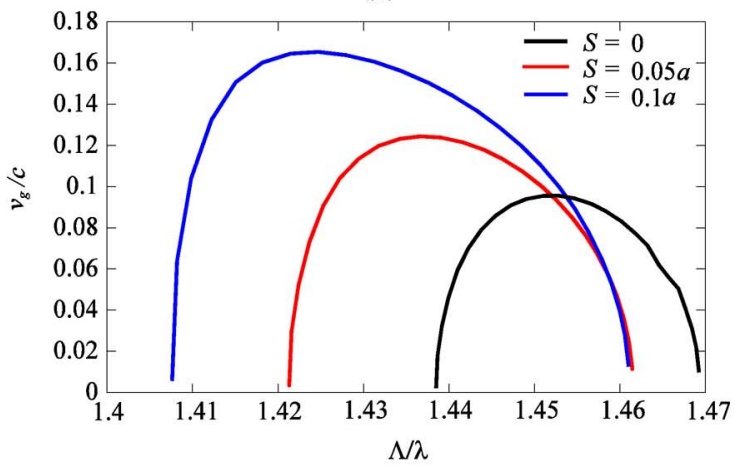

(c)

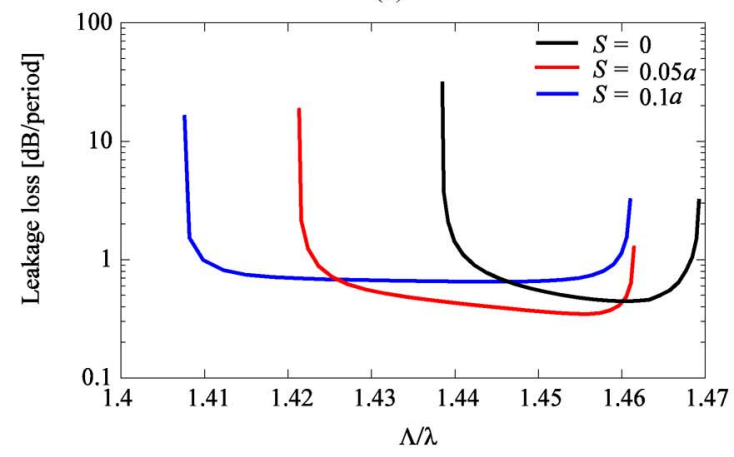

(d)

Fig. 3. (a) Structure of 1-D PC-CROW with shifted air-holes, (b) dispersion curves, (c) group velocity, and (d) leakage losses of 1-D PC-CROW for $S=$ $0,0.05 a$, and $0.1 a$.

light stronger in the cavity than silica cladding CROW. Leakage loss is decreased to about $0.33 \mathrm{~dB}$ per period. This leakage loss can be further reduced by applying previously introduced technique. For example, we consider air-bridge 1-D PC-CROW with changed air-hole radius neighboring the cavities. In this case, we 


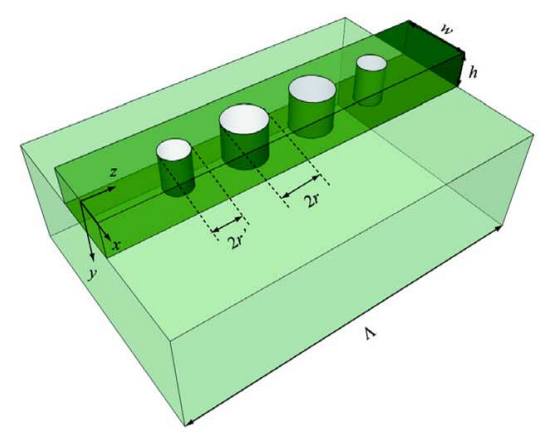

(a)

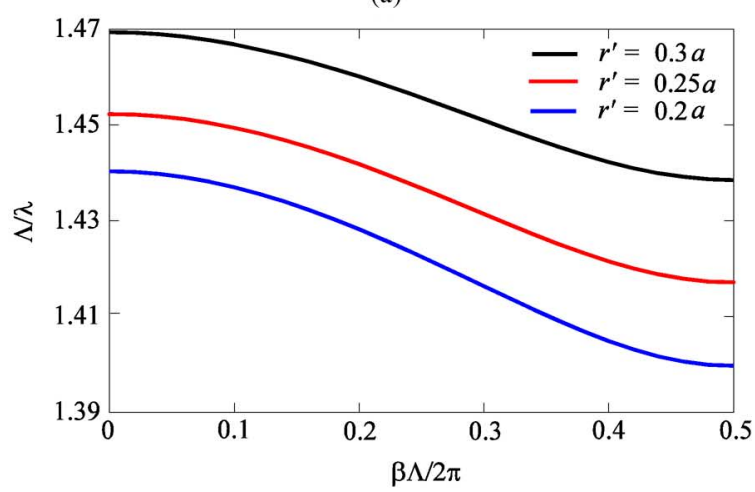

(b)

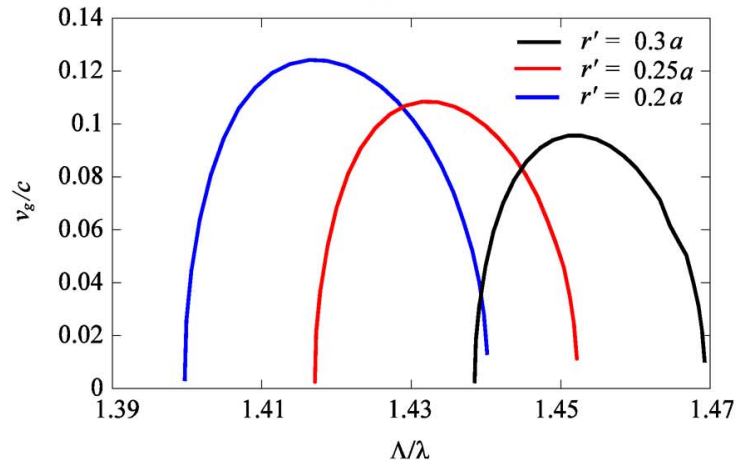

(c)

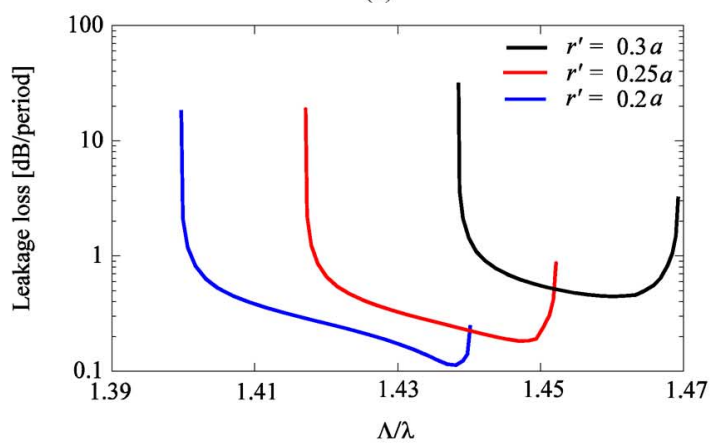

(d)

Fig. 4. (a) Structure of 1-D PC-CROW with changed size of air-hole, (b) dispersion curves, (c) group velocity, and (d) leakage losses of 1-D PC-CROW for $r^{\prime}=0.3 a, 0.25 a, 0.2 a$.

set $r^{\prime}$ as $0.2 a$. Blue curves in Figs. 5(b), (c), and (d) show dispersion curve, group velocity, and leakage loss, respectively, for air-bridge 1-D PC-CROW with changed air-hole radius. We can see that the leakage loss is reduced to $0.023 \mathrm{~dB}$ per period and normalized group velocity is 0.105 . Air-bridge 1-D PC-CROW

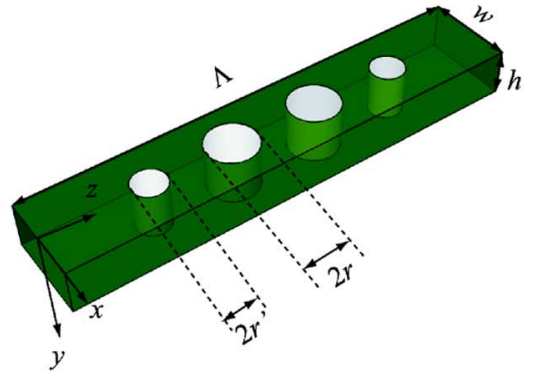

(a)

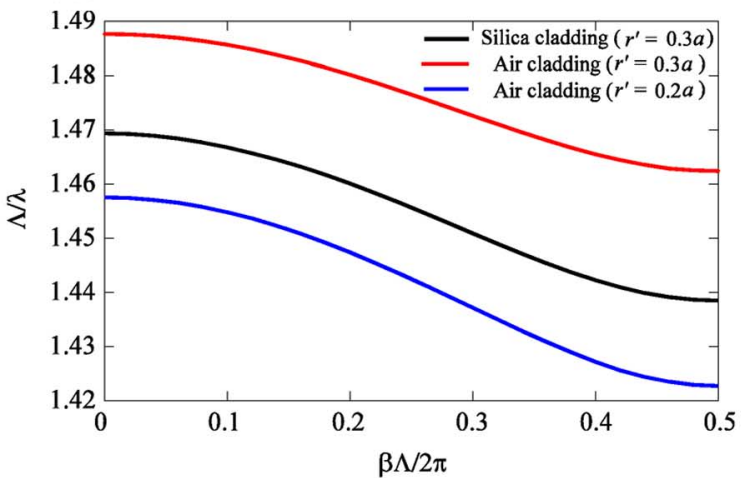

(b)

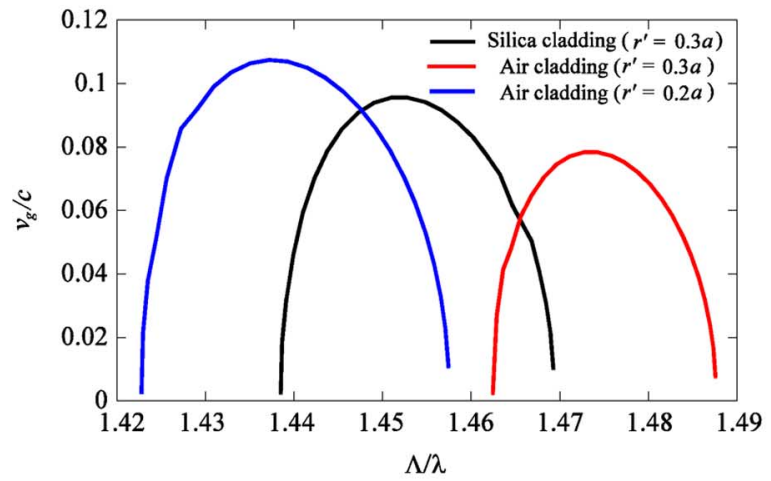

(c)



(d)

Fig. 5. (a) Structure. (b) dispersion curves, (c) group velocity, and (d) leakage losses of air-bridge 1-D PC-CROW.

can achieve small group velocity and low-loss characteristics simultaneously, however, we note that simplicity, which is advantage of 1-D PC-CROW, is lost in air-bridge 1-D PC-CROW because some additional fabrication processes are needed. 


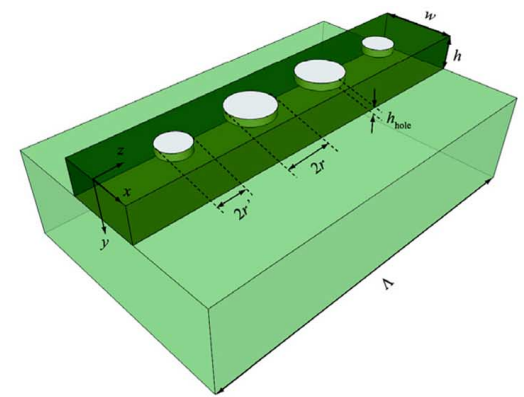

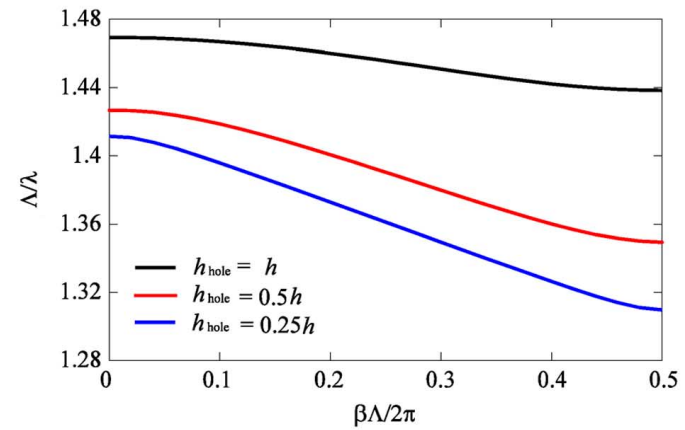

(b)

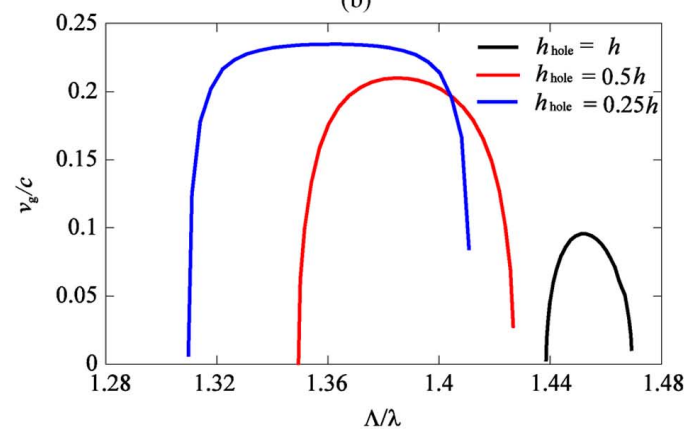

(c)

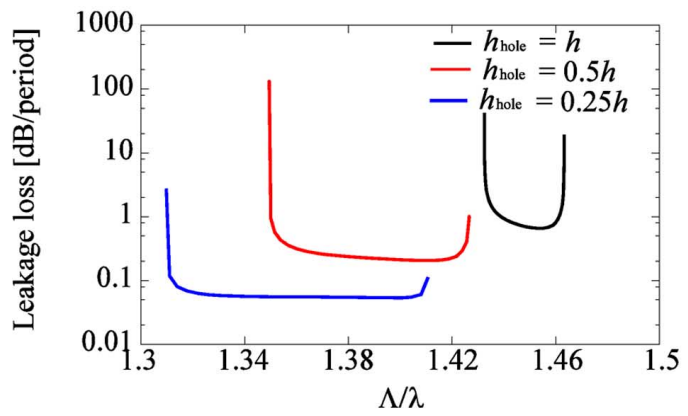

(d) (a)



(e)

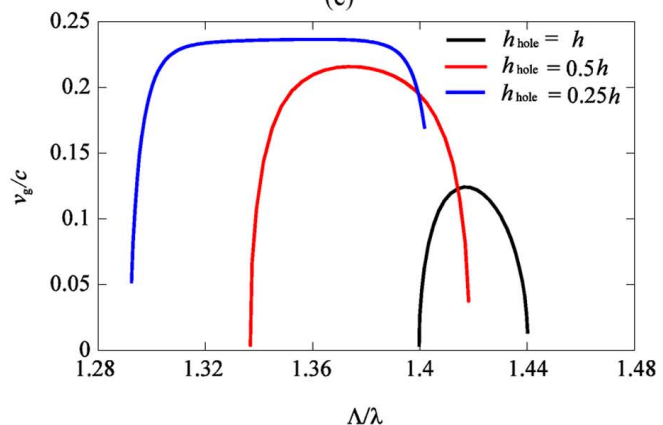

(f)

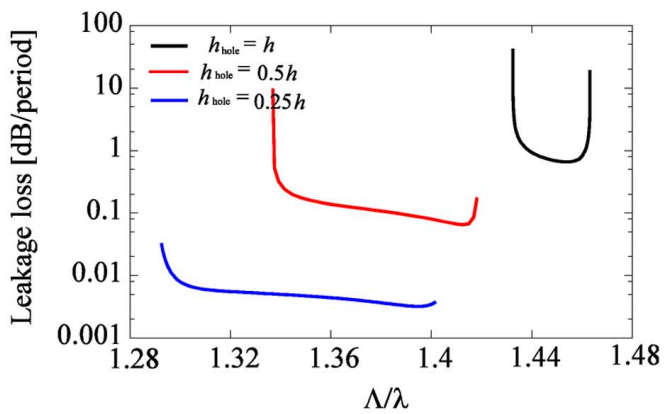

(g)

Fig. 6. (a) Structure of 1-D PC-CROW with shallow etched air-hole, (b) dispersion curves, (c) group velocity, (d) leakage losses of 1-D PC-CROW for $r^{\prime}=0.3 a$, (e) dispersion curves, (f) group velocity, and (g) leakage losses of 1-D PC-CROW for $r^{\prime}=0.2 a$.

\section{1-D PC-CROW With Shallow Etched Air-Hole}

The another method to reduce the leakage loss is weakening the refractive index modulation of CROW. It is expected that such structures can realize small leakage loss, however, group velocity becomes larger. If one assumes to apply CROW to pulse delay devices, leakage loss should be as small as possible even if group velocity is not small because delay time becomes longer by multiplying resonators. In order to weaken the refractive index modulation, we consider 1-D PC-CROW with shallow etched air-hole as shown in Fig. 6(a). We set air-hole

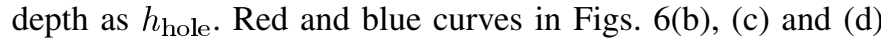
show dispersion curves, group velocity, and leakage losses of 1-D PC-CROW for $h_{\text {hole }}=0.5 h$ and $0.25 h$, respectively. Black curves show those of normal 1-D PC-CROW. We note that the air-hole radius are set as $r=r^{\prime}=0.3 a$. We can see that by reducing the air-hole depth, the dispersion curve shifts to lower frequency region and the group velocity becomes larger. When we choose $h_{\text {hole as }} 0.25 h$, leakage loss is reduced to $0.07 \mathrm{~dB}$ per period. Next, in order to achieve much lower leakage loss structure, we set $r^{\prime}$ as 0.2a. Red and blue curves in Figs. 6(e), (f) and 
(g) show dispersion curves, group velocity, and leakage losses of 1-D PC-CROW for $h_{\text {hole }}=0.5 h$ and $0.25 h$, respectively $\left(r^{\prime}=0.2 a\right)$. If we set $r^{\prime}=0.2 a$ and $h_{\text {hole }}=0.25 h$, leakage loss can be reduced about 2 orders of magnitude lower compared with normal structure $\left(h_{\text {hole }}=h\right.$ and $\left.r^{\prime}=r=0.3 a\right)$. However, we note that this structure has also problem in terms of simplicity in fabrication process as air-bridge structure.

\section{CONCLUSION}

We have evaluated leakage losses of 1-D PC-CROW by using 3 -D vector FEM for periodic structure analysis. We have shown that the leakage losses are quite high especially for vertical direction, however, it was shown that the leakage losses can be reduced by adjusting the air-hole position and radius neighboring cavities. We have also shown that the leakage losses are reduced from $0.5 \mathrm{~dB}$ per one period to $0.023 \mathrm{~dB}$ per one period by applying air-bridge structure to 1-D PC-CROW with changed air-hole radius. Moreover, we have proposed 1-D PC-CROW with shallow etched air-hole and shown that the leakage loss can be decreased about 2 orders of magnitude lower compared with normal structure. Since such structure's modulation of refractive index is weak, group velocity becomes larger. Air-bridge 1-D PC-CROW or 1-D PC-CROW with shallow etched air-hole can achieve low-loss characteristics, however, additional fabrication processes may be needed. Therefore, designing small group velocity and low-loss 1-D PC-CROW with simple structure is next issue.

\section{REFERENCES}

[1] T. Sakamoto, A. Okada, M. Hirayama, Y. Sasaki, O. Moriwaki, I. Ogawa, R. Sato, K. Noguchi, and M. Matsuoka, "Optical packet synchronizer using wavelength and space switching," IEEE Photon. Technol. Lett., vol. 14, pp. 1360-1362, Sep. 2002.

[2] E. Kuramochi, T. Tanabe, and M. Notomi, "Large-scale arrays of ultrahigh- $Q$ coupled nanocavities," Nat. Photon., vol. 2, pp. 741-747, Nov. 2008.

[3] A. Melloni, A. Canciamilla, C. Ferrari, F. Morichetti, L. O'Faolain, T. F. Krauss, R. De La Rue, A. Samarelli, and M. Sorel, "Tunable delay lines in silicon photonics: Coupled resonators and photonic crystals, a comparison," IEEE Photon. J., vol. 2, pp. 181-194, Apr. 2010.

[4] H. J. S. Dorren, M. T. Hill, Y. Liu, N. Calabretta, A. Srivatsa, F. M. Huijskens, H. de Waardt, and G. D. Khoe, "Optical packet switching and buffering by using all-optical signal processing methods," J. Lightw. Technol., vol. 21, pp. 2-12, Jan. 2003.

[5] J. Leuthold, P. A. Besse, E. Gamper, M. Dulk, S. Fischer, G. Guekos, and H. Melchior, "All-optical Mach-Zender interferometer wavelength converters and switches with integrated data- and control-signal separation scheme," J. Lightw. Technol., vol. 17, pp. 1056-1066, Jun. 1999.

[6] J. Company, J. Cascon, D. Peter, and B. Ortega, "Reconfigurable fiberoptic delay line filters incorporating electrooptic and electroabsorption modulators," IEEE Photon. Technol. Lett., vol. 11, pp. 1174-1176, Sep. 1999.

[7] N. Stefanou and A. Modinos, "Impurity bands in photonic insulators," Phys. Rev. B, vol. 57, pp. 12127-12133, May 1998.

[8] A. Yariv, Y. Xu, R. K. Lee, and A. Scherer, "Coupled-resonator optical waveguide: A proposal and analysis," Opt. Lett., vol. 24, pp. 711-713, Jun. 1999.

[9] M. Bayindit, B. Temelkuran, and E. Ozbay, "Propagation of photons by hopping: A waveguiding mechanism through localized coupled cavities in three-dimensional photonic crystals," Phys. Rev. B, vol. 61, pp. R11855-R11857, May 2000.
[10] S. Oliver, C. Smith, M. Rattier, H. Benisty, C. Weisbuch, T. Krauss, R. Houdre, and U. Oesterle, "Miniband transmission in a photonic crystal coupled-resonator optical waveguide," Opt. Lett., vol. 26, pp. 1019-1021, Jul. 2001.

[11] S. Lan, S. Nishikawa, H. Ishikawa, and O. Wada, "Engineering photonic crystal impurity bands for waveguides, all-optical switches and optical delay lines," IEICE Trans. Electron., vol. E85-C, pp. 181-189, Jan. 2002.

[12] S. Mookherjea and A. Yariv, "Coupled-resonator optical waveguides," IEEE J. Sel. Top. Quantum Electron., vol. 8, pp. 448-456, May 2002.

[13] T. J. Karle, D. H. Brown, R. Wilson, M. Steer, and T. F. Krauss, "Planar photonic crystal coupled cavity waveguides," IEEE J. Sel. Top. Quantum Electron., vol. 8, pp. 909-918, Aug. 2002.

[14] T. J. Karle, Y. J. Chai, C. N. Morgan, I. H. White, and T. F. Krauss, "Observation of pulse compression in photonic crystal coupled cavity waveguides," J. Lightw. Technol., vol. 22, pp. 514-519, Feb. 2004.

[15] J. K. S. Poon, J. Scheuer, Y. Xu, and A. Yariv, "Designing coupledresonator optical waveguide delay lines," J. Opt. Soc. Am. B, vol. 21, pp. 1665-1673, Sep. 2004

[16] J. K. Poon, L. Zhu, G. A. Derose, and A. Yariv, "Transmission and group delay of microring coupled-resonator optical waveguides," Opt. Lett., vol. 31, pp. 456-458, Feb. 2006.

[17] F. Xia, L. Sekaric, M. O'Boyle, and Y. Vlasov, "Coupled resonator optical waveguides based on silicon-on-insulator photonic wires," Appl. Phys. Lett., vol. 89, pp. 041122-041124, Jul. 2006.

[18] Y. Xu, R. K. Lee, and A. Yariv, "Propagaion and second-harmonic generation of electromagnetic waves in a coupled-resonator optical waveguide," J. Opt. Soc. Amer. B, vol. 17, pp. 387-400, Mar. 2000.

[19] W. J. Kim, W. Kuang, and J. O'Brien, "Dispersion characteristics of photonic crystal coupled resonator optical waveguides," Opt. Exp., vol. 11, pp. 3431-3437, Dec. 2003.

[20] M. L. Povinelli and S. Fan, "Radiation loss of coupled-resonator optical waveguides in photonic-crystal slabs," Appl. Phys. Lett., vol. 89, pp. 191114-191116, Nov. 2006.

[21] Y. Sugimoto, S. Lan, S. Nishikawa, N. Ikeda, H. Ishikawa, and K. Asakawa, "Design and fabrication of impurity band-based photonic crystal waveguides for optical delay lines," Appl. Phys. Lett., vol. 81, pp. 1946-1948, Sep. 2002.

[22] S. Nishikawa, S. Lan, N. Ikeda, Y. Sugimoto, H. Ishikawa, and K. Asakawa, "Optical characterization of photonic crystal delay lines based on one-dimensional coupled defects," Opt. Lett., vol. 27, pp. 2079-2081, Dec. 2002.

[23] P. Velha, E. Picard, T. Charvolin, E. Hadji, J. Rodier, P. Lalanne, and D. Peyrade, "Ultra-high Q/V-Fabry-Perot microcavity on SOI substrate," Opt. Exp., vol. 15, pp. 16090-16096, Nov. 2007.

[24] A. R. Md Zain, N. P. Johnson, M. Sorel, and R. M. De La Rue, "High quality-factor 1-D-suspended photonic crystal/photonic wire silicon waveguide mico-cavities," IEEE Photon. Technol. Lett., vol. 21, pp. 1789-1791, Dec. 2009.

[25] A. Martinez, J. Garcia, P. Sanchis, F. C. Soto, J. Blasco, and J. Marti, "Intrinsic losses of coupled-cavity waveguides in planar-photonic crystals," Opt. Lett., vol. 32, pp. 635-637, Mar. 2007.

[26] Y. Kawaguchi, N. Kono, K. Saitoh, and M. Koshiba, "Loss reduction mechanism for coupled cavity waveguides in one-dimensional photonic crystals," J. Lightw. Technol., vol. 26, pp. 3461-3467, Oct. 2008.

[27] N. Kono and M. Koshiba, "Three-dimensional finite element analysis of nonreciprocal phase shifts in magneto-photonic crystal waveguides," Opt. Exp., vol. 13, pp. 9155-9166, Nov. 2005.

[28] O. Schenk and K. Gatrner, "Solving unsymmetric sparse systems of linear equations with PARDISO," J. Fut. Gen. Comput. Syst., vol. 27, pp. 4032-4035, Nov. 1991.

[29] K. C. Huang, E. Lidorikis, X. Jiang, J. D. Joannopoulos, and K. A. Nelson, "Nature of lossy bloch states in polaritonic photonic crystals," Phys. Rev.B, vol. 69, pp. 195111-1-195111-10, May 2004.

Yuki Kawaguchi (S'10) was born in Hokkaido, Japan, on November 15, 1983. He received the B.S. degree in electronic engineering and the M.S. degree in media and network technologies from Hokkaido University, Sapporo, Japan, in 2007 and 2009, respectively, where he is currently working toward the Ph.D. degree in information science.

He has been a Research Fellow of the Japan Society for the Promotion of Science since 2009. He has been engaged in research on modeling of photonic integrated circuit using finite element method. Mr. Kawaguchi is a member of the Institute of Electronics, Information and Communication Engineers (IEICE) of Japan. 
Kunimasa Saitoh (S'00-M'01) was born in Hokkaido, Japan. He received the B.S., M.S., and Ph.D. degrees in electronic engineering from Hokkaido University, Sapporo, Japan, in 1997, 1999, and 2001, respectively.

From 1999 to 2001, he was a Research Fellow of the Japan Society for the Promotion of Science. From 2001 to 2005, he was a Research Associate of Graduate School of Engineering at Hokkaido University. In 2005, he became an Associate Professor at Graduate School of Information Science and Technology, Hokkaido University. He has been engaged in research on fiber optics, nano-photonics, integrated optical devices, and computer-aided design and modeling of guided-wave devices using finite element method, beam propagation method, and so on. He is an author or coauthor of more than 100 research papers in refereed international journals.

Prof. Saitoh is a member of the Institute of Electronics, Information and Communication Engineers (IEICE) and the Optical Society of America (OSA). In 1999 and 2002, he was awarded the Excellent Paper Award and the Young Scientist Award from the IEICE, respectively, and in 2008, the Young Scientists' Prize of the Commendation for Science and Technology from the Ministry of Education, Culture, Sports, Science, and Technology (MEXT), Government of Japan.
Masanori Koshiba (M'76-SM'87-F'03) was born in Sapporo, Japan. He received the B.S., M.S., and Ph.D. degrees in electronic engineering from Hokkaido University, Sapporo, Japan, in 1971, 1973, and 1976, respectively.

In 1976, he joined the Department of Electronic Engineering, Kitami Institute of Technology, Kitami, Japan. From 1979 to 1987, he was an Associate Professor of Electronic Engineering at Hokkaido University, and in 1987, he became a Professor there. He has been engaged in research on wave electronics, including microwaves, millimeter-waves, lightwaves, surface acoustic waves (SAW), magnetostatic waves (MSW), and electron waves, and computeraided design and modeling of guided-wave devices using finite element method, boundary element method, beam propagation method, and so on. He is an author or coauthor of more than 300 research papers in refereed international journals. $\mathrm{He}$ is an author of books Optical Waveguide Analysis (New York: McGraw-Hill, 1992) and Optical Waveguide Theory by the Finite Element Method (Tokyo, Japan: KTK Scientific/Dordrecht, The Netherlands: Kluwer Academic, 1992), and is a coauthor of the books Analysis Methods for Electromagnetic Wave Problems (Boston, MA: Artech House, 1990), Analysis Methods for Electromagnetic Wave Problems, Vol. Two (Boston, MA: Artech House, 1996), Ultrafast and Ultra-parallel Optoelectronics (Chichester, U.K.: Wiley, 1995), and Finite Element Software for Microwave Engineering (New York: Wiley, 1996).

Prof. Koshiba is a fellow of the Institute of Electronics, Information and Communication Engineers (IEICE), and is a member of the Institute of Electrical Engineers of Japan, and the Institute of Image Information and Television Engineers of Japan. In 1987, 1997, and 1999, he was awarded the Excellent Paper Awards from the IEICE, in 1998, the Electronics Award from the IEICE-Electronics Society, and in 2004, the Achievement Award from the IEICE. From 1999 to 2000, he served as a President of the IEICE-Electronics Society, and in 2002, he served as a Chair of the IEEE-LEOS (Lasers and Electro-Optics Society) Japan Chapter. Since 2009, he has served as a Vice-President of the IEICE and a Chair of the IEEE Sapporo Section. 